\title{
Research and Design of Online Education Platform Based on Named Data Network
}

\author{
Linglin $\mathrm{Li}^{1,}$, , Dening Jiang ${ }^{1, b,{ }^{*}}$, Di Miao ${ }^{1, c}$ Kaixin $\mathrm{Li}^{1, \mathrm{~d}}$ \\ ${ }^{1}$ School of Electronic Engineering, Tianjin University of Technology and Education, Tianjin, China \\ *Corresponding author: Dening Jiang (jdn9999@163.com) \\ a453094036@qq.com, bjdn9999@163.com, '09111042@bjtu.edu.cn, d582297205@qq.com
}

Keywords: NDN, online education, Mini-Net

\begin{abstract}
This paper proposes a new method for data transmission using the Named Data Network (NDN) architecture, and introduces the new NDN network into online education innovatively. Based on the information center network (ICN), the special advantages of the NDN network are utilized to improve the real-time transmission of the online education network. This paper first builds an Ubuntu-based system platform, simulates network data transmission behavior using Mini-NDN simulation experiments, and uses data analysis methods to analyze the network delay of real-time transmission of online education courses. We theoretically analyze the transmission characteristics of the NDN architecture and experimentally verify the superiority of its network transmission. Data analysis and experimental results show that the adoption of NDN network in online education has the advantages of small delay and high real-time performance.
\end{abstract}

\section{Introduction}

At present, the construction and research of online education platform is mainly based on the development and application of APP and PC, and has achieved phased results. The online education model continuously promotes the perfect combination of Internet technology and education, enhances the technical level of the education platform, and realizes the optimal sharing of educational resources. So far, whether it is based on APP or PC-based online education, all data transmission is single-wave transmission, and it is easy to generate blocking waves during transmission, which affects the timeliness and real-time of data transmission. Finding a new network transmission method and network protocol has become an urgent problem in the field of online education technology. In response to the above problems, research scholars have proposed a content-centric future network[2-3]. The NDN (Named Data Networking) [4] network architecture adopted in this paper is based on a content-centric future network and redesigned the network concept. NDN abandons the transmission mechanism of traditional networks and establishes a new network model to fundamentally solve the above problems, and has higher adaptability and better performance.

\section{Online education forwarding process based on NDN}

The architecture of NDN. NDN has good compatibility, and it can be compatible with the current IP network architecture, and on this basis, the current network system is optimized. It retains the advantages of the current TCP/IP network architecture, retains the slim waist architecture[5], and in order to facilitate the naming of data objects, the IP address of the data node is no longer used as the transmission basis. However, this thin waist structure is different from the thin waist structure of the IP architecture, in which the IP address is replaced by a content block in the thin waist portion. In the communication process of the NDN network, each transmission content has its own unique name. The name becomes the only feature of data request, recovery and forwarding, which enables reliable and transparent distribution of content and improves resource utilization in the network[6]. And redesigned the routing protocol, set up new interest packages and data packets, and finally reset the 
security authentication method based on the content. These designs and changes can fundamentally improve the shortcomings of the IP network architecture.

Node structure and package type of NDN. Communication in the NDN is driven by the content request of the data requester. Each node in the network contains three data structures as shown in Figure 1. The three data structures are: (1) Recording data forwarding interface PIT (Pending Interest Table), (2) Forwarding Information Base (FIB) (Forwarding Information Base) (3) Content Storage CS (Content Store).

In the online education data transmission based on the NDN network architecture, two kinds of protocol transmission packages are set: Interest packet and Data packet [8]. As shown in Figure 1, the user side needs to send the Interest package request when there is a need for the course. The nodes or content sources in the network will base on the specific conditions of their CS, PIT and FIB choose to send the Data package directly to the client according to the original path or forward the request.

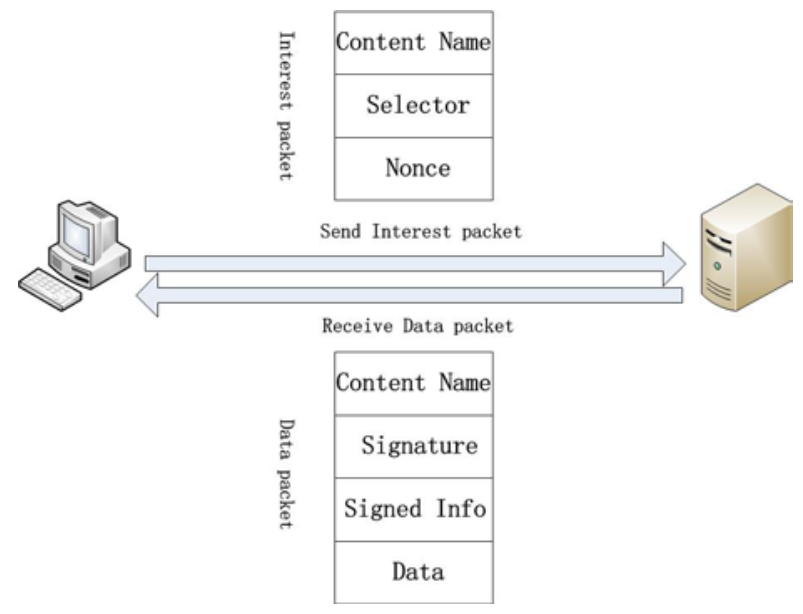

Fig1. The structure and transmission diagram

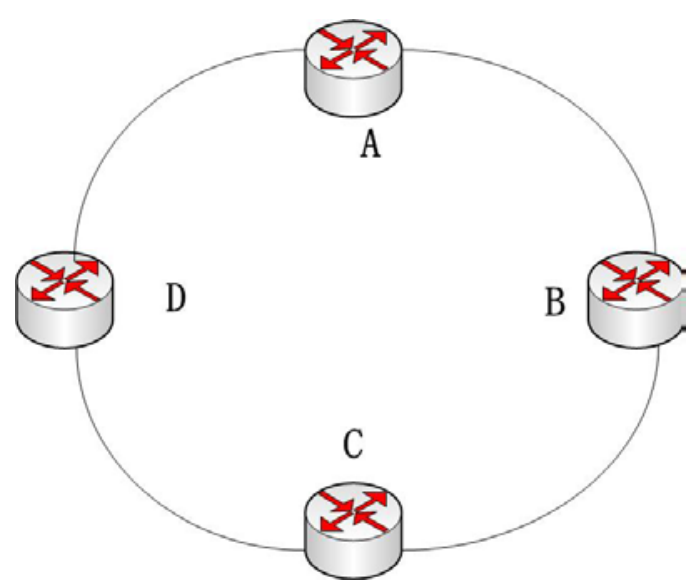

Fig.2 Network topology

process of interest package and packet.

Online education real-time forwarding process in NDN network. In NDN network, network data forwarding is mainly motivated by data requester, and its request forwarding data is based on interest packets and data packets. When the client needs to request an online real time course, the client node will send an Interest package with the required online real-time course name to the NDN network. In the network, the routing nodes that receive an Interest package with a class name will look for their own CS tables, PIT tables and FIB tables in turn. Then decide how to deal with the online real-time course request interest package.

First, when any network node receives the Interest package containing the course name, (1) the node first queries in the CS table. If a copy of the requested course content is found in the table, the course content is obtained from CS and a Data package is created. Then, the node sends the Data packet to the client along the reverse path of the interest package, and discards the Interest packet. (2) If the course content or copy is not queried in the CS table, the query is made in the PIT table. If a record of the required course content is found in the PIT, it indicates that the node has forwarded the Interest package request for the same course content. The node simply adds the destination interface of such an Interest packet to the corresponding PIT entry to avoid the meaningless repeated forwarding of the Interest packet. (3) If the requested course content information cannot be found in the PIT table, it indicates that the received Interest packet is forwarded for the first time. Therefore, you need to query the FIB table to find the next appropriate interface to forward the Interest packet. At the same time, the project entry of the course content is added in the PIT table, and the name and destination interface of the requested course content are recorded. (4) If the relevant forwarding path cannot be found in the FIB table, the Interest packet is discarded. 
Second, when the content source receives the Interest package containing the course name, you can extract the requested course content directly from the database to create a data package,and discard the Interest packet and then send the Data packet along its reverse path.

Finally, when the intermediate node receives the Data packet, then query CS first. If the data packet is found, discard the data packet directly. Otherwise, according to the caching policy, it is judged whether the course content needs to be cached to the node. Then query the PIT table of the node to determine the destination interface of the Data packet and forward it, and delete the corresponding PIT entry after the Data packet is forwarded.

\section{The ping test between nodes}

NDN experimental platform construction. In order to realize the online education real-time transmission system experiment described in this paper, it is first necessary to install the Mini-NDN simulation actual operating environment in the Linux system. To simulate a multi-host test environment, we use a virtual machine to implement the operating system installation. The bridge environment is used to configure the network environment so that hosts can communicate with each other. Based on this, the underlying operating environment of the NDN is built and configured. This article mainly configures the core components: a. Named Data Networking Forwarding Daemon, b. Link library (ndn-cxx), c. NSLR.

Network Topology. This experiment uses a four-node ring topology of the gateway to perform experiments, as shown in Figure 2 above. A, B, C, and D are respectively four nodes in the NDN network, and the ring connection is used as the connection mode. The ring network topology has better real-time performance and short cable length. Using light as a communication material can improve the network speed and enhance the anti-interference ability. This structure adapts to the transmission characteristics of online education and the need for the network, enabling experiments to simulate real-world networks more realistically. Therefore, this paper uses a ring topology to conduct experiments.

Analysis of results. In order to realize the real-time transmission function of online education so that it can be effectively utilized on the set routing nodes, we studied the forwarding strategy of NFD and modified the key data. First of all, we use the official tool component of ndn-tools in Mini-NDN, which mainly applies the ping test package accessibility. By default, when the NFD is initially started, it will register with the ndn:/localhost/nfd/rib entry in the FIB. Without increasing the initial startup of the FIB entry, the client can obtain status data returned by the native routing node by sending an interest package for the online course. As shown in Figure 3. 


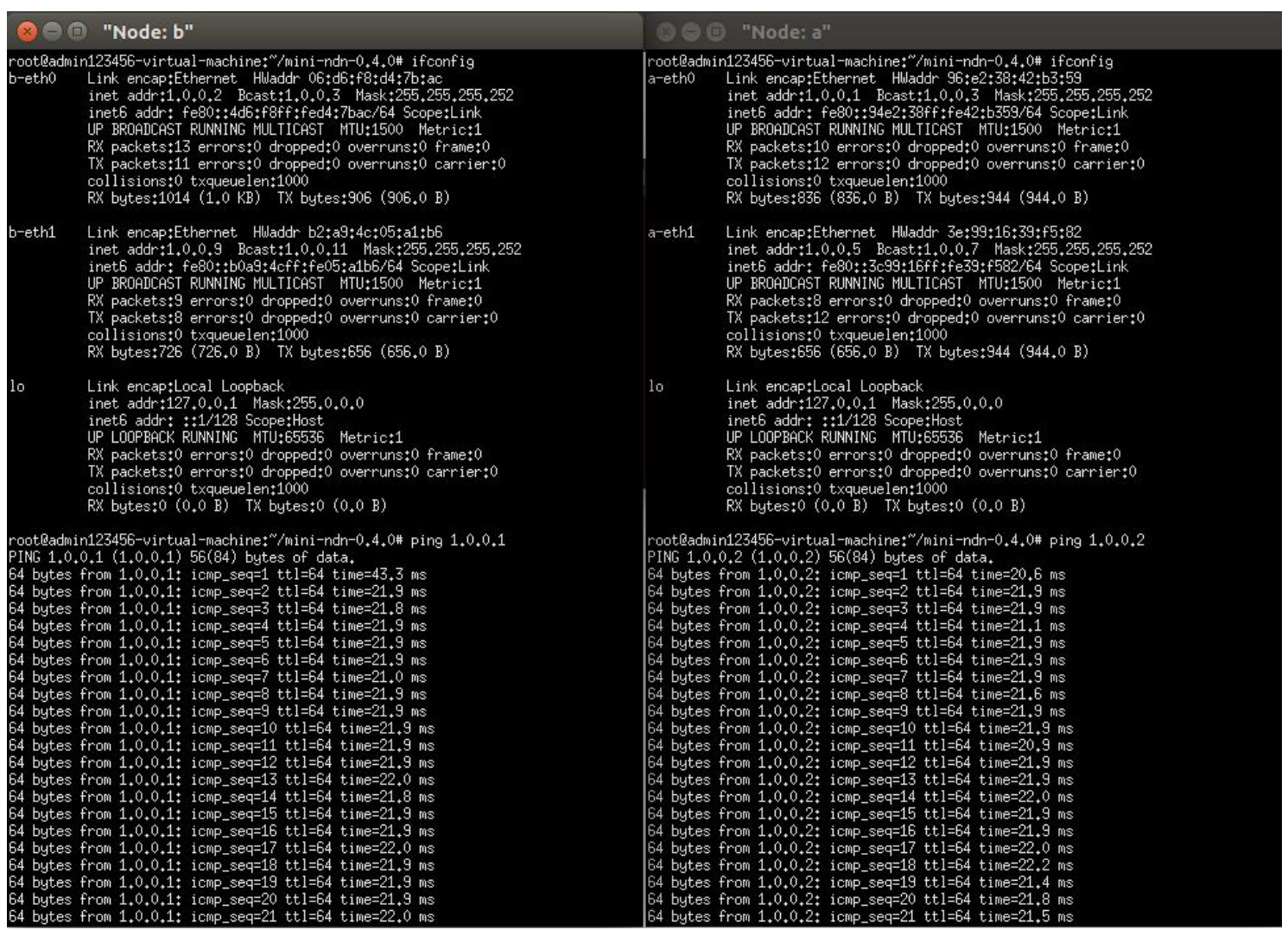

Fig.3 Node Ping map

Through the above experiments, we can see that the network architecture using the data naming network can successfully ping the network and complete the online education real-time data transmission function, so that the purpose of real-time transmission of online education data can be smoothly operated. Therefore, the research work at the application layer can focus on the realization of logic, so that the network function can be further improved, and at the same time further improve the efficiency of the network to obtain online education real-time courses.

\section{Conclusions and prospects}

With the continuous development of Internet technology, the IP network architecture will eventually be replaced. The named data network architecture is about to become the trend of the future network. This paper proposes a new method of data transmission using a named data network architecture, and introduces a new type of NDN network into online education innovatively. NDN based on the information center network (ICN). This paper makes use of the transmission advantages of the NDN network to achieve the purpose of improving the real-time nature of the online education network. This paper first builds the Ubuntu system platform, and uses the Mini-NDN simulation experiment to simulate the real-time transmission situation of the network. Then based on the experimental data, the network delay in the real-time transmission process of the online education network is analyzed. The results show that the named data network shows higher performance in the process of transmitting real-time data and network resources. Named data networks have outstanding performance in terms of transmission speed, efficiency, and security and stability.

\section{Acknowledgements}

This work was supported by Tianjin Natural Science Foundation (17KPHDSF00290, 16KPHDSF00050), Tianjin Science and Technology Project (17JCTPJC48300, 16ZXHLSF00200), Tianjin Education Commission Scientific Research Plan Project (JWK1606), Tianjin University of Technology and Education talent start-up project (KYQ01614), Tianjin Education Science "13th 
Five-Year Plan" Plan(VEYP5044), University Student Innovation Training Program (201810066056).

\section{References}

[1] V Jacobson, DK Smetters, JD Thornton, et al, in: Networking named content, edited by Proceedings of the 5th international conference on Emerging experiments and technologies, Rome, December 1-4, 2009. ACM Press. 2009, pp. 1-12

[2] C Yi, A Afanasyev, L Wang, et al, in: Adaptive forwarding in named data networking, edited by Acm Sigcomm Computer Communication Review, 2012, 42(3), pp.62-67

[3] Information on http://minindn.memphis.edu

[4] L Zhang, Deborah Estrin, Jeffrey Burke, et al, in Name data networking(NDN) Project, edited by PARC Technical Report NDN-0001, 2010

[5] X Jiang, J Bi, Y Wang, et al, in: An easy matrix computation based simulator of ndn, edited by 2012 Third International Conference on Networking and Distributed Computing. IEEE, 2012, pp. 36-39

[6] L Zhang, A Afanasyev, J Burke, et al, in: Named data networking, edited by Acm Sigcomm Computer Communication Review, 2014, 44(3), pp. 66-73

[7] M Meisel, V Pappas, L Zhang, in: Ad hoc networking via named data, edited by Proceedings of the fifth ACM international workshop on Mobility in the evolving Internet architecture. ACM, 2010, pp. 3-8

[8] A Afanasyev, J Shi, B Zhang, et al, “NFD developer’s guide”, NDN Technical Report NDN-0021, 2015 